\title{
Game theory based Ad-hoc On Demand Distance Vector Routing Protocol to Extend the Wireless Sensor Networks Life Time
}

\author{
M.Premkumar', M.P.Chitra ${ }^{2}$, X.Alisha Celestin ${ }^{3}$, T.Kausalya ${ }^{4}$, M.N.Nandhini Priya ${ }^{5}$ \\ ${ }^{1-5}$ Department of Electronics and Communication Engineering, Panimalar Institute of Technology,Chennai-600123,Tamil \\ Nadu, India
}

\begin{tabular}{l}
\hline \hline Article Info \\
\hline Article history: \\
Received Nov 30, 2018 \\
Revised Apr 30, 2019 \\
Accepted Aug 15, 2019 \\
\hline
\end{tabular}

\section{Keyword:}

Game theory

Ad-hoc on demand distance

vector routing

Lifetime

Wireless Sensor Network

\begin{abstract}
This paper proposes a solution with an objective to increase the lifetime of wireless sensor networks (WSNs) via a concept of game theory enabled adhoc on demand distance vector (AODV) routing protocol. Game theory is an optimal promising candidate for decision making in a wireless networking scenario to find the optimal path for data packets transfer between source node and destination node, where combination with the AODV routing algorithm, a procedure of game theory enabled AODV (GTEAODV) is developed and proposed in this research paper. The developed and proposed methodology is validated through simulation in NS2 environment and the results show an improvement in energy lifetime of the order of 30-35\% in comparison to the existing routing methodology which uses co-operative routing techniques among the nodes in WSN. Further, the throughput of game theory enabled adhoc on demand routing is also highly improved in comparison to existing traditional approaches though obtained results. Though, game theory approach is an existing approach concatenation of it with AODV can provide increased network performance which is significant as portrayed in research results shown in the paper. Hence, by virtue of providing enhanced energy lifetime and data security through the nature of the algorithm, the proposed GTEAODV algorithm can be employed in defence applications for secure data transmission and reception for forthcoming deployment of 5G systems which are blossoming in world wide scenario.
\end{abstract}

Copyright (C) 2019 Institute of Advanced Engineering and Science. All rights reserved.

\section{Corresponding Author:}

M. Premkumar,

Department of Electronics and Communication Engineering,

Panimalar Institute of Technology,

Chennai-600123, Tamil Nadu, India

Email: ermpremkumar@yahoo.co.in

\section{INTRODUCTION}

As the era of information and communication technology (ICT) progresses towards the 2020, where $5 \mathrm{G}$ technology[1],[2] in terms of data rate of $1 \mathrm{Gbps}$ is expected to be catered practically, data collection, dissemination and processing from various nodes deems to be essential. In applications such as military [3], monitoring of environments, agriculture sector, manufacturing in industrial units, medical health care for humanity, sensors gain prime importance and the operation in a network perspective is highly beneficial to the society globally. Network of sensor nodes as represented by universality as wireless sensor networks (WSN) collect data from distributed sensor nodes for monitoring and recording the physical conditions of the environment and organizing the collected data at a central location. WSN operation defines wireless connectivity and formation of network spontaneously, such that data from the sensor are broadcasted[4] and they are wirelessly transmitted. As in WSN, the nodes are energy constrained which implies energy consumption [5] are powered by the batteries, where it is complicated to have updation or charging its battery 
for two main reasons. The foremost fact is that the batteries cost are expensive and it takes more time to recycle. The second fact is that the need to improve energy efficiency to procrastinate the lifetime of network as it is an important issue in wireless sensor networks.

There are many solutions proposed to optimize the energy consumption in conventional WSN but the protocol loses the data during the transfer of information packets. Various research papers have been proposed with protocols such as destination sequenced distance vector (DSDV) routing [6], dynamic source routing(DSR), ad-hoc on demand distance vector (AODV) [7],[8] with energy efficiency and secured routing pertaining to energy consumption. The important aspect in the above enlisted routing protocols is that during transmission of a bulk amount of data from source node to destination node in a straight line approach there is a chance of more packet loss. To reduce the packet loss ratio, more number of nodes are established between the coverage area of source node and destination node where it splits the data into the packets according to the path identified with its internet protocol (IP) address. At the centre of the coverage area, the data which splits already will get merged at the nodes and then it reaches the destination. However, routing loop problem and there are promising routing protocols such as destination sequenced distance vector (DSDV), dynamic source routing (DSR) and ad-hoc on demand distance vector (AODV) are used. Among the protocols DSDV routing is a table-driven routing scheme, where it requires large memory space, high connection delay setup and obviously consumes more energy whereby making it not preferrable for optimal operation of WSN. The other option, DSR is a protocol which employs an approach in which it is reactive and elimination of requirement to flood the network periodically with the table update messages is not needed. In a reactive ondemand approach, a route is established only on requirement and finding routes to all other nodes in the network by table driven approach is not essentially required. But the route maintenance mechanism of DSR protocol does not locally repair a broken link and the performance degrades rapidly with increasing mobility. Hence, due to the pros and cons of the DSDV and DSR routing protocols, ad-hoc on demand distance vector routing (AODV) routing [9],[10] is proposed in which each packet holds the source address and destination address and information about routers through which they pass, and in addition exhibit, compact memory size and also consumes less energy.

Several research papers are proposed for assessing the energy lifetime and efficiency of sensor nodes. Cooperative information transmission with weighted power allocation with consideration of certain parameters such as node energy, overhead transmission a cooperative routing algorithm is proposed in [11]. Joint cooperative routing with minimal collision for wireless sensor networks with flow of information in multiple forms is given in the literature of [12] which is prominent for WSN scenario. The research work presented in [13] addresses combination of lifetime and transport delay for wireless sensor networks with a suitable constraint on reliability. WSN are subjected to various attacks relating to security in order to encounter that [14] provides an active detection security technique. Industry based wireless sensor networks are provided with energy efficient scheme which uses the method of adjustable broadcasting in radius to improve network upgradation [15]. For software defined networking based wireless sensor networks, fuzzy incorporated discovery protocol is employed which increases the lifetime and decreases the packet loss ratio [16]. RLMan, energy management algorithm using reinforcement learning a novel approach is presented in [17]. Data transmission from sensor to base station, using greedy routing procedure with smallest transmission power for achieving delivery of packets in WSN is depicted in literature[18]. All the above literatures provide excellent works for energy consumption and lifetime for WSN to a great extent. In order to further enhance the energy lifetime this paper proposes combination of a well known decision making concept Game Theory[19] along with ad-hoc on demand distance vector (AODV) routing. Concatenation of game theory to AODV routing methodology can provide effective decision making for information flow of data packets from source node to destination node, secure efficient routing] which infers optimal operation of WSN thereby contributing to enhanced energy lifetime which make it profitable for data transmission and reception. This approach can also be used for WSN based applications.

The research paper is presented as follows. Section I presents the introductory information related to the research paper, section II presents gametheory based AODV routing from source node to destination node. Section III portrays the simulation procedure in game theory based AODV routing methodology for enhanced energy lifetime. Section IV presents the simulation results for various parameters under consideration. Section $\mathrm{V}$ concludes the research paper. 
2. PROPOSED SYSTEM- GAME THEORY BASED AODV ROUTING

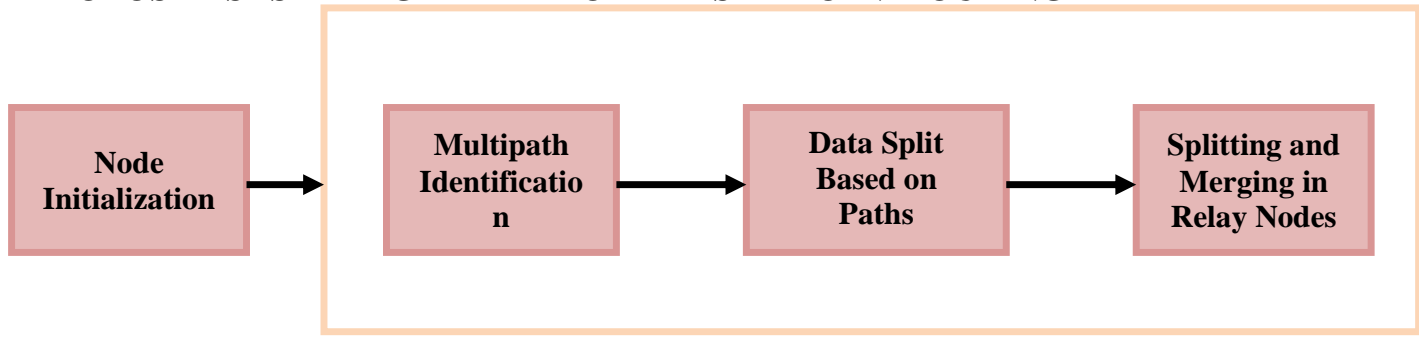

Figure 1. Block Schematic of Game Theory

Game theory[20],[21] is a decision making concept applied normally to select different routes to transfer the data from transmitting node (or) source node to receiving node (or) destination node. Game theory is, multiagent decision theory and it is a mathematical model that defines utilizing and maximizing agents whose actions affect one another's utilities. In this research paper, ad-hoc on demand distance vector (AODV) procedure is used where the nodes are initialized along with multipath identification as in Figure 1. The concept is that the internet protocol (IP) address of the source node, destination node and the routers through which it passes to reach the destination node are also embedded along with the data in the packets. The information stored in the source is splitted and merged based on the algorithm and the whole information is transmitted through the routing path with reference to the destination IP address. The packets size at the central nodes are very small due to the division and inspite of loss of packets, it does not affect the whole information. Figure 2 shows transmission of data through nodes and Table 1 shows the simulation testbed considered.

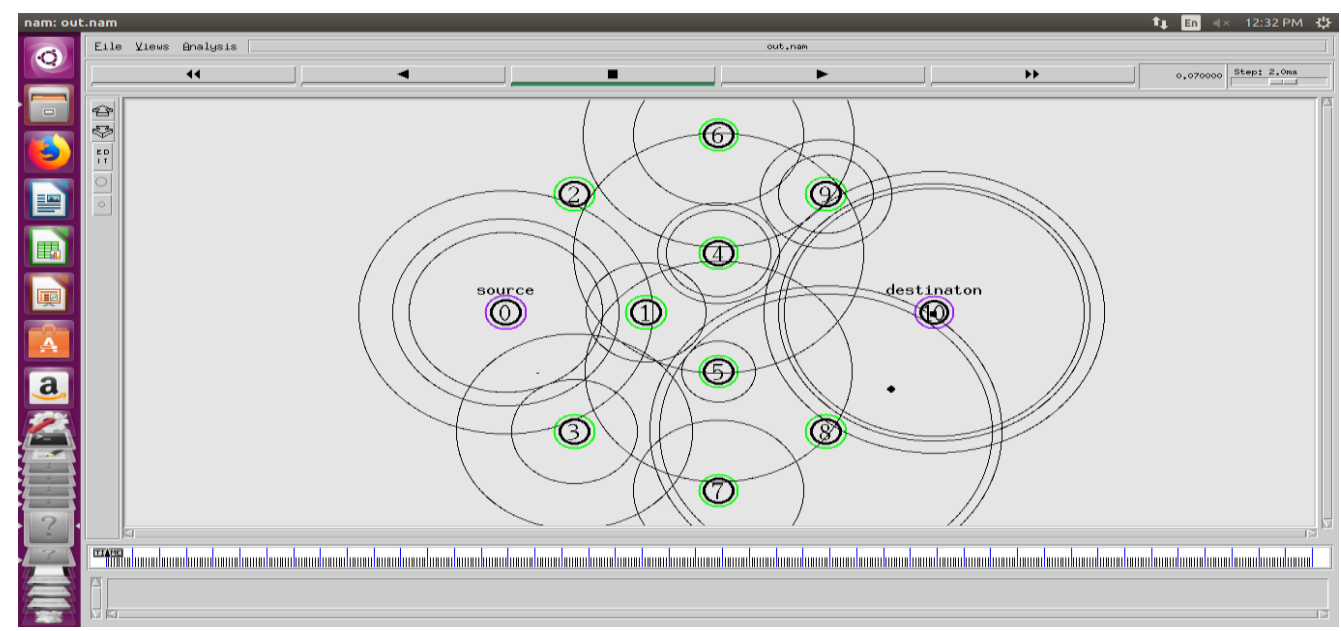

Figure 2. Transmission of data through the nodes

Table 1. Simulation Test Bed in NS2

\begin{tabular}{cc}
\hline Parameters in Testbed & Values for Testbed \\
\hline Area of simulation & $(500$ X 500)m \\
Number of nodes & 11 \\
Types of Routing Protocol & AODV \\
Internet Protocol type & UDP \\
Antenna model & Omni directional \\
Maximum number of Packets & 100 \\
Type of MAC & 802.11 \\
Transmission Speed & $300 \mathrm{Mbps}$ \\
Bandwidth & $2.4 \mathrm{GHz}$ \\
Security Algorithm & AODV \\
Source traffic & $\mathrm{CBR}$ \\
Payload & 500 \\
\hline
\end{tabular}




\section{METHODOLOGY SIMULATION PROCEDURE IN GAMETHEORY}

Ad-hoc on demand distance vector is a routing protocol designated for mobile ad hoc networks (MANETs) and it is used in zigbee oriented low power, low data rate wireless ad hoc network. Basically when a node that wants to transfer data packet to other node that is not in its range,it determines a route through other nodes. AODV routing operates by route request message (RREQ) and route reply message (RREP). If a node is not confined in a range with the other node, it needs to talk to the node to send a RREQ and this RREQ contains source internet protocol (IP) address, sequence number and destination IP address, sequence number, as well as the lifespan of the RREQ. Rebroadcasting of RREQ must be applied, if a neighbour donot possses the knowledge how to route to a destination node. The RREP message is sent to the source, if a neighbour only knows a route towards the destination node. Figure 3 shows operation of AODV routing protocol.

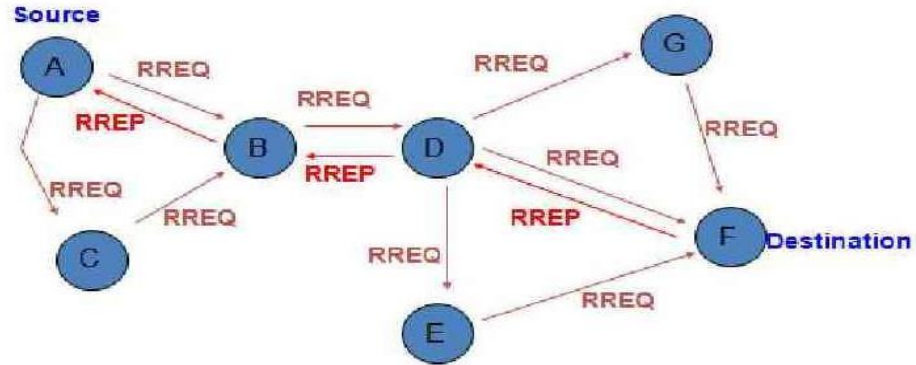

Figure 3. AODV routing protocol

The operating procedure is that whenever a source node such as A intends to transmit a data pakcet to a destination node $\mathrm{F}$ and if it does not know an intended route, then a procedure to find out path known as path discovery process is done to find the respective node. A request (RREQ) packet is broadcasted to its neighbors, which forwards the information request so along as destination node or an intermediate node $\mathrm{B}$ with a fresh route is being determined. An individual sequence number is maintained in each node along with a broadcast ID, where it is updated for each and every RREQ where a node initiates along with internet protocol (IP) address. Further the source node includes where it has most recent sequence number for the destination node. Nodes which are intermediate communicate with RREQ if a route is available for the intended sequence number which is on par with the value available in RREQ. On course of RREQ forwarding, intermediate nodes update in their routing tables the neighbor address from where the broadcasted packet is received so a reverse path can be established. Moreover, when ample number of similar RREQ are received later, such packets are discarded instantly. On reception of the RREQ when it reaches the destination node $F$ or in an intermediate node such as $\mathrm{D}$ with a new route they respond by sending a route reply (RREP) pakcet to the neighbor which originated the first RREQ. When RREP is routed in reverse direction, nodes have forward routing direction in their tables which specifies the node from which RREP has arrived. The presence of an active forward route is indicated through forward route entries and with each route entry a route timer will remove the data if it is not done within a specific duration of time. As long as a source node A moves in the direction and if it reinitiates a route discovery procedure a new path to destination $\mathrm{F}$ is foud out which is depicted in Figure 3 . This process is done along as a new route is necessary to be found out. Figure 4 presents a flowchart of an AODV protocol.

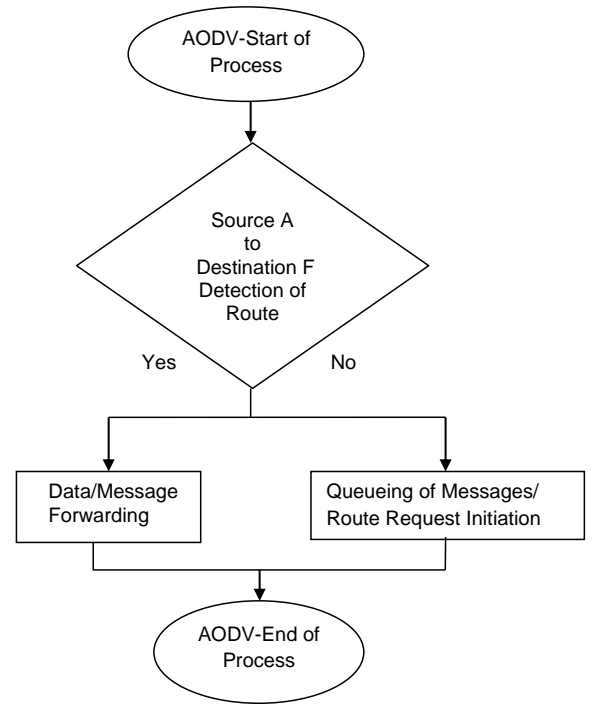

Figure 4. Operation Sequence of AODV Protocol 


\section{METHODOLOGY SIMULATION PROCEDURE IN GAMETHEORY}

In this research paper, using Network Simulator 2(NS2) software, the performance metrics and the behavior of nodes which are created virtually are analyzed. NS2 is an open source simulation tool which runs on Linux and Ubuntu16.04 OS. There are five steps for simulation process which are: (i) Initialization, (ii) Object creation, (iii)Traffic generation, (iv)Finish procedure, and (v)Traffic call.

\section{Step 1 - Initialization}

Channel is the fundamental entity for the transmission of data. In this, the transmission takes place through the wireless medium. First, we declare the wireless channel a flat fading channel in which the information is intended to travel. To predict the path loss between the transmitting antenna and receiving antenna, generally two ray ground propagation model is used. As per the open systems interconnection (OSI) model, physical layer carries information to any medium either in analog or digital form and provides links to wireless physical layer standards such as wireless local area networks(WLAN), wireless microwave access for interoperability (WiMAX), fixed orthogonal frequency division multiplexing (OFDM), global system for mobile (GSM) communications and code division multiple access(CDMA). The medium access control(MAC) layer provides physical connection to the network among several computers since it is one of the sub layers of data link control layer and to manage the traffic occuring during the transmission, queuing mechanism is employed. Antenna used at the transmitters and receivers, omni directional antenna is preferred which radiates the radio frequency uniformly in all directions. In addition to that, the number of nodes, queue size, packet size and the AODV protocol is initialized. In order to place the nodes XY region is declared and transmission start and stop time is also given. The transmission is started with the energy of 100 joules.

\section{Step 2 - Object creation}

Object creation is necessary to run the program. Generally network animator (NAM) and TRACE files are created. In the TRACE file, the data are given, which holds the location of nodes within the boundary, number of packets distributed, the start time, stop time and also calls the functions which are initialized. Topology object is created within the topological boundary to keep the track of movement of mobile nodes within them. The data in the TRACE file is moved to the ANIMATOR file and then it is possible to view the output, network simulation traces and packet trace data virtually.

\section{Step 3 - Traffic generation}

For the node movement, a node movement file is inbuilt in NS2 simulator. This file can be had from NS2 along with other node movement files. In addition to node movements, traffic flows between setup mobile nodes, are also available in traffic pattern file. Within the mobile nodes, the data packets are sent, forwarded or received by the nodes within the hearing range of one another.

\section{Step 4 - Finish procedure}

The finish procedure closes the trace file and starts the network animator(NAM) file. To run the simulator, object is used to execute the finish procedure after the given simulation time. The run command will start the simulation. Whenever execution is completed, CMU Trace output file and NAM output file is obtained. In the NAM window, mobile nodes move virtually.

\section{Step 5 - Traffic call}

In traffic call process, a userdatagram protocol (UDP) connection is setup between two mobile nodes. In order to synchronize the data between the UDP agents and the corresponding destination, constant bit rate (CBR) agent is used. The main purpose of the traffic call is to call the UDP and CBR agents and after performing all the above functions the simulation begins. When the simulation ends, it is necessary to define the stop time and inform mobile nodes to reset internal network components. Finally the stop procedure is called to flush out traces and to close the trace file. Before flush out, the data in the trace file is moved to animator file, and it is possible to view the transmission of data virtually.

\section{SIMULATION RESULTS}

In this section, simulation results are portrayed for wireless sensor networks (WSNs) scenario having 11 nodes with area under simulation (500X500)m using AODV routing algorithm, considering 100 packets for data transfer between a source node and a destination node. The parameters considered to analyze the performance of game theory enabled AODV algorithm in WSN are throughput[22], end to end delay in WSN, packet delivery ratio in WSN and packet loss ratio in wireless sensor networks. The significant contribution of this research paper is to provide enhanced energy lifetime for WSN in comparison to the existing traditional procedure of co-operative routing procedure. 
Throughput is a performance metric for successful message delivery rate over a communication channel. It is calculated using Alfred Aho Peter Weinberger and Brian Kernighan (AWK) script, which processes the trace file and produces the result. Throughput is the ratio of the number of bytes received over the current time instance[23].

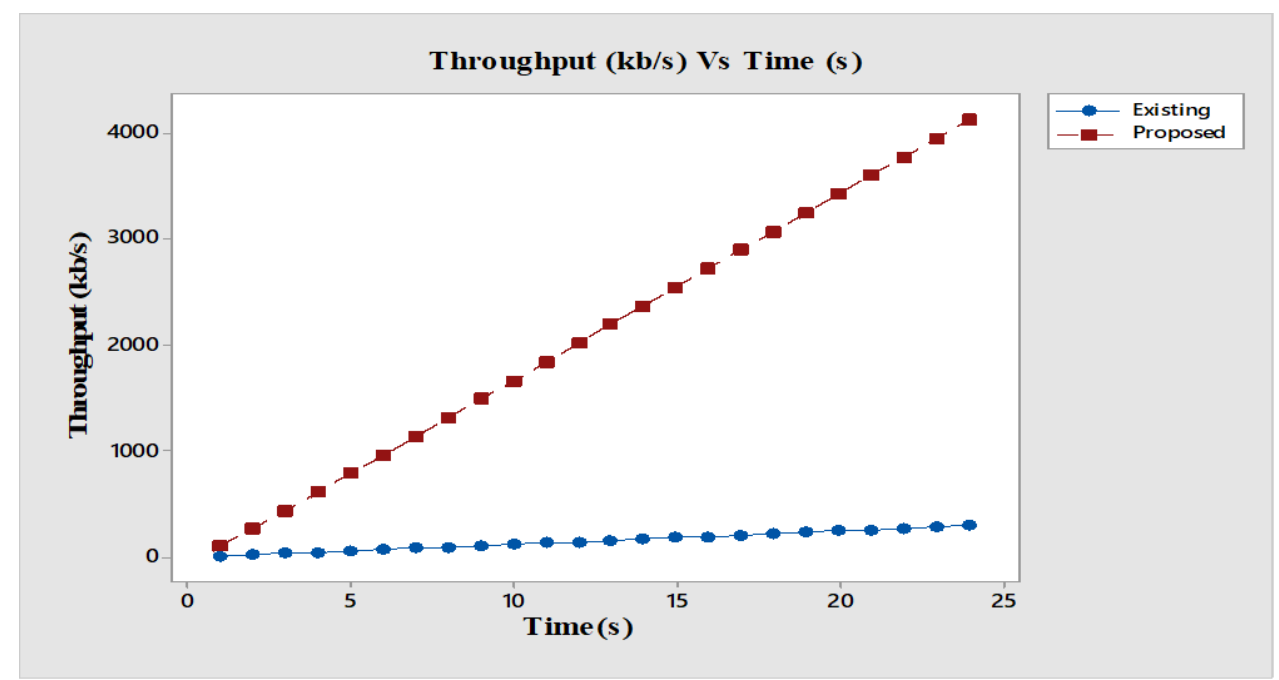

Figure 5. WSN Throughput Result Against Time

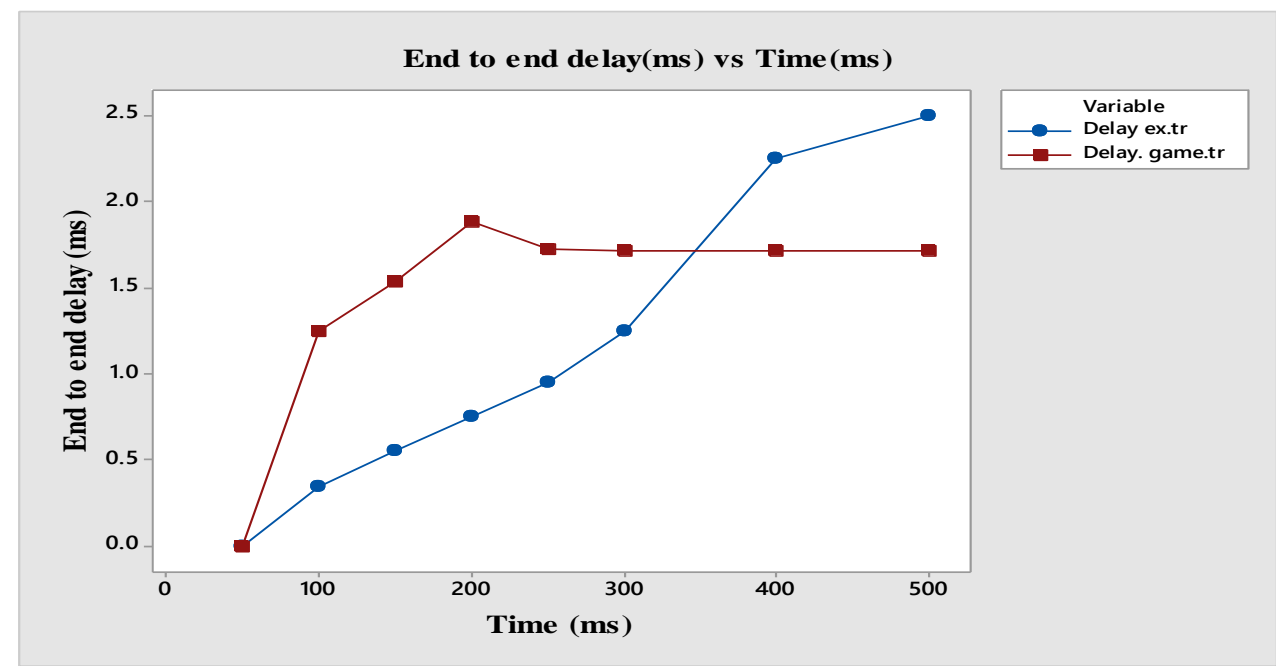

Figure 6. WSN End to End Delay Against Time

Figure 5 shows the throughput of WSN using Game theory [24] enabled AODV algorithm and the existing cooperative routing-based algorithm. The proposed GTEAODV algorithm provides higher throughput of the order of $4000 \mathrm{~kb} / \mathrm{s}$ as time progress for data transfer of packets in the WSN, whereas existing algorithm provides very minimal throughput as time progresses.

Similarly, end to end delay against time is also observed as in Figure 6, the difference between the time at which the sender sends the data packets and time at which the receiver receives the packet constitutes end to end delay. The result is calculated using AWK script which process the trace file. Formula for calculating delay is, that delay is the difference of end time and start time. Figure 6 shows the end to end delay of WSN using Game theory enabled AODV algorithm. The proposed GTEAODV algorithm provides lower end to end delay of the order of $1.8 \mathrm{~ms}$ as time progress for data transfer of packets in the WSN in comparison to cooperative routing-based algorithm.

Figure 7 shows the packet delivery ratio performance based on the packets received at the destination node on par with the packets generated at the source. Using AWK script, trace file is processed and the result is produced. Packet delivery ratio is the ratio of received packets to the sequence number. Figure 7 , shows the packet delivery ratio of WSN using game theory enabled AODV algorithm. The proposed GTEAODV algorithm provides maximum packet delivery ratio of $58 \%$ as time progress for successful data transfer of packets in the WSN[25] in comparison to cooperative routing algorithm. 


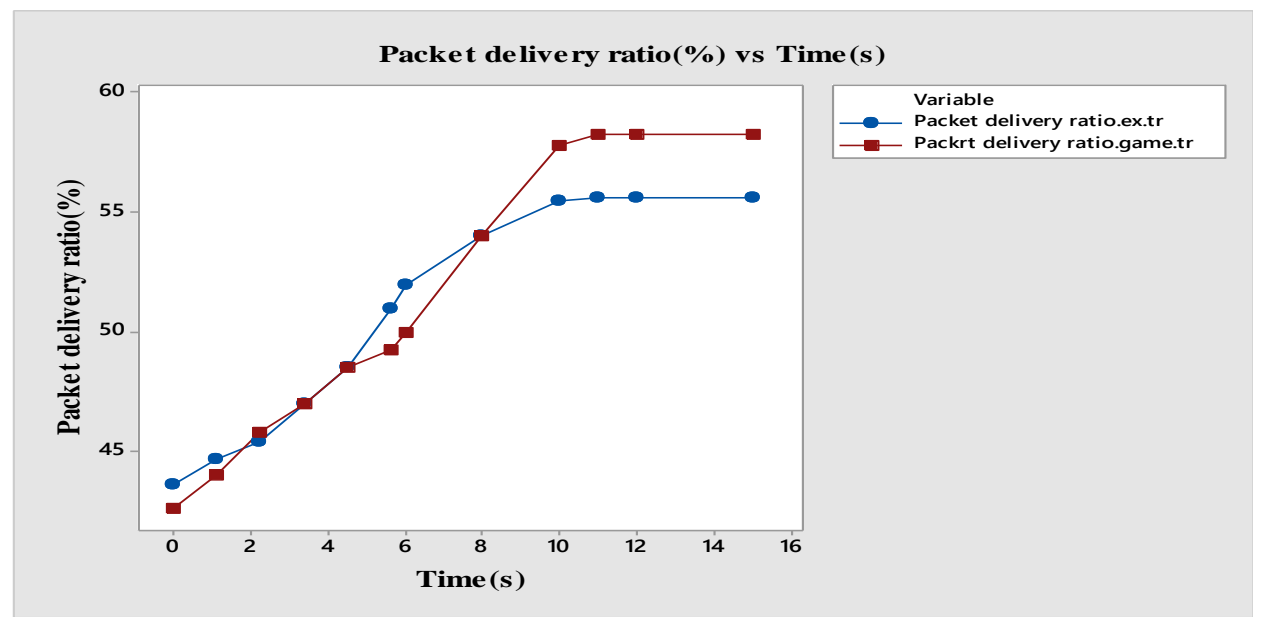

Figure 7. WSN Packet Delivery Ratio Against Time

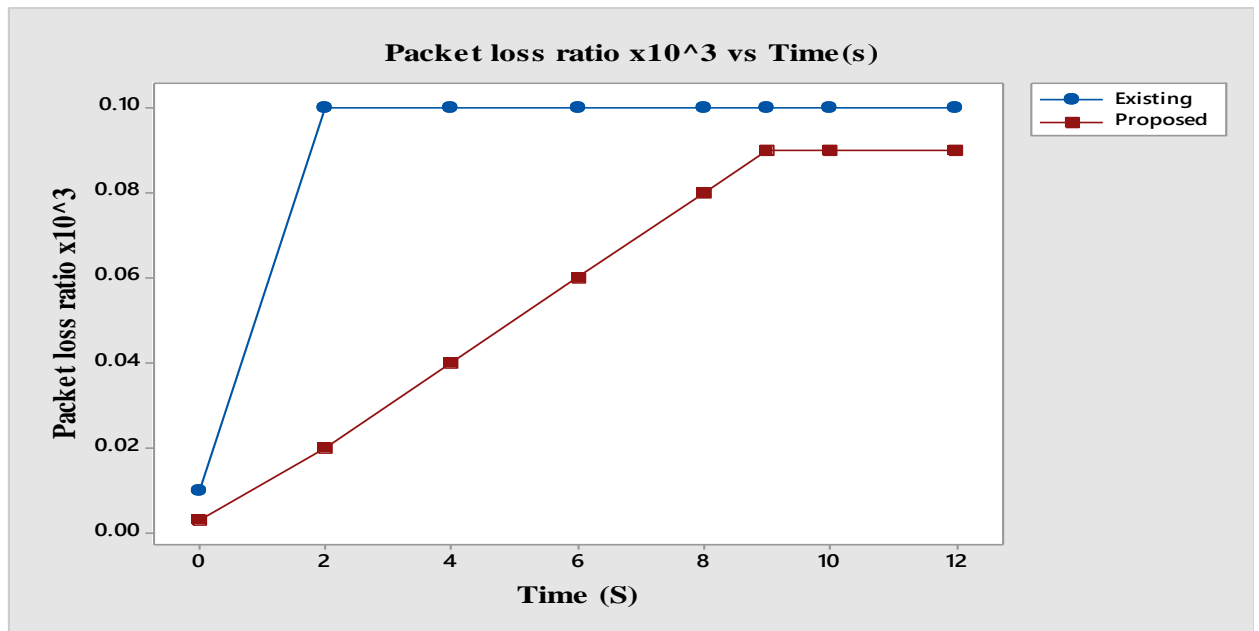

Figure 8. WSN Packet Loss Ratio Against Time

Figure 8 shows the packet loss ratio of WSN using game theory enabled AODV algorithm. The proposed GTEAODV algorithm provides minimum packet loss ratio as time progress for data transfer of packets in the WSN. In comparison, packet loss ratio for existing cooperative routing method is very high of the order of 0.10 as time progresses during execution. Packet loss ratio is obtained by calculating the difference between the generated and the received packets. To obtain this AWK script processes the trace file and produces the results. Packet loss $=$ Generated packets - received packets

Figure 9 shows the energy lifetime of WSN[25] using game theory enabled AODV algorithm. The proposed GTEAODV algorithm provides maximizes the energy lifetime of the order of $42 \mathrm{~mJ}$ within a short span of time on start of the operation itself contrary to existing algorithm which takes $20 \mathrm{~mJ}$. This infers that gametheory based AODV protocol provides optimal performance due to decision making than that of cooperative routing. The WSN[26] energy remains in the nodes at the destination side constitutes the residual energy. It is also obtained by calculating the difference between the initial energy and the consumed energy at the nodes. Residual energy = Initial energy of the nodes - energy consumed by the nodes. This can be very useful in applications such as green monitoring [27], power control[28] pertaining to wireless sensor networks. Further, lifetime is an important performance determining metric in WSN as portrayed in [29],[30] which are based on game theoretic approaches. On the whole the results obtained and presented in this research paper can be used for energy harvesting[31] in wireless sensor networks, low latency requirement for wireless sensor networks[32] and also for health care related scenarios of wireless sensor networks[33]. 


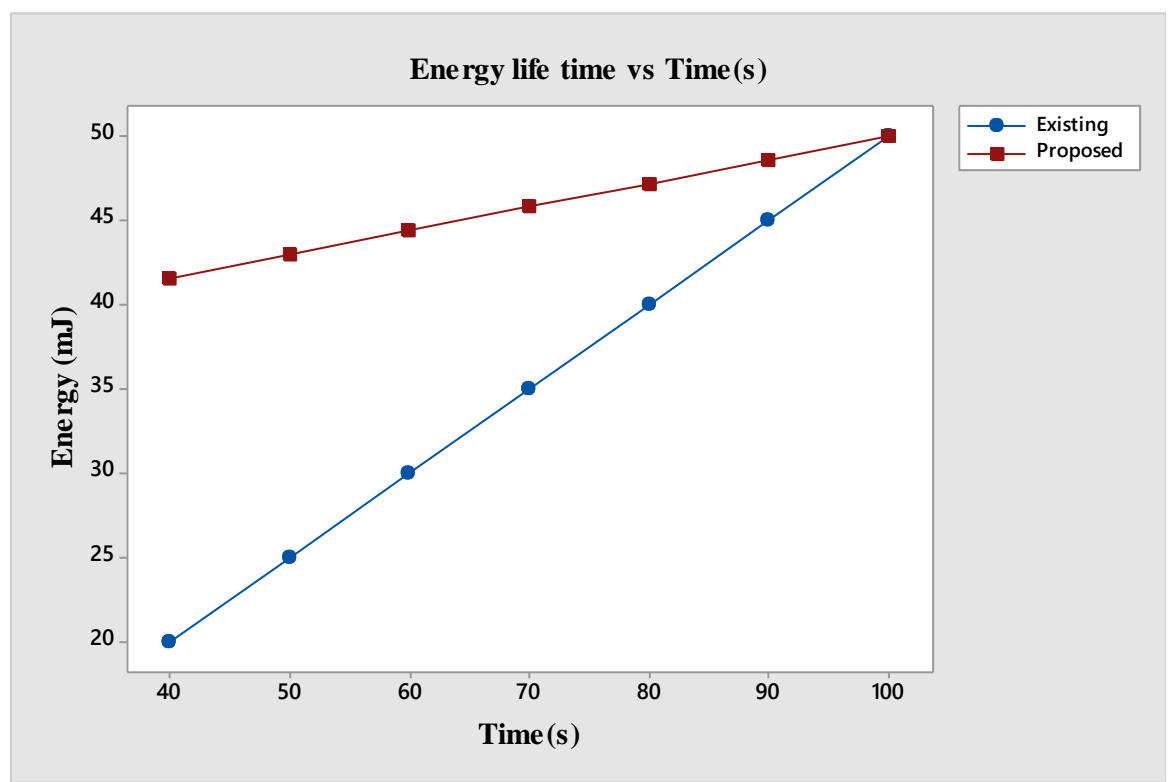

Figure 9. WSN Energy Against Time

\section{CONCLUSION}

In this paper, ad-hoc on demand distance vector (AODV) routing protocol is proposed, based on gametheory. With intelligent decision making due to game theory, transmission of data from the source node to destination node is secured throughout the routing protocol due to the usage of AODV. The algorithm chooses optimal node set which is cooperative, balances energy cost for transmitters, and computes error during transmission of information and chooses the alternative path to reach the destination. This algorithm maximizes 30-35\% of energy of the sensor nodes which can be significant for ICT based systems intended to be developed for $5 \mathrm{G}$ systems. As a future extension of this research paper the implementation aspects could be realized pertaining to designing of WSN, data acquisition and interpretation which will be beneficial for researchers working in domain of Wireless Sensor Networks.

\section{REFERENCES}

[1] P. Lynggaard and K.E. Skouby, “Deploying 5G Technologies in Smart City and Smart Home Wireless Sensor Networks with Interferences", Wireless Personal Communications, 2015; 81(4): 1399-1413.

[2] R. Li, Z. Zhao, X. Zhou, G. Ding, Y. Chen, Z. Wang and H. Zhang "Intelligent 5G: When Cellular Networks Meet Artificial Intelligence", IEEE Transactions on Wireless Communications, 2017; 24 (5):.175-183.

[3] S. Renubala and K. S. Dhanalakshmi, "Trust based secure routing protocol using fuzzy logic in wireless sensor networks", in Proc. of. IEEE International Conference on Computational Intelligence and Computing Research (ICCIC), Coimbatore, India, 2014: 1-5.

[4] K. Dewangan and S. Bhattacharjee, "Lifetime maximizing cooperative energy efficient broadcast tree in multi hop wireless networks", in Proc. of. 2014 IEEE International, Conference on Advance Computing Conference (IACC), Gurgaon, India 2014: 318-322.

[5] M. Sajid, K. Khan, U. Qasim, A. Khan, S. Tariq, and N. Javaid, "A New Linear Cluster Handling (LCH) Technique Toward's Energy Efficiency in Linear WSNs," 29th IEEE International Conference on Advanced. Information Networking and Applications (AINA), 2015,Gwajgju, South Korea: 389-393.

[6] R. Ennaji and M. Boulmalf, "Routing in Wireless Sensor Networks", in Proc. of.International Conference on Multimedia Computing and Systems (ICMCS'09), Quarazatte, Morocco2009: 495-500.

[7] K. P. Ng and C. Tsimenidis, "Energy-balanced dynamic source routing protocol for wireless sensor network", in Proc. of. IEEE Conference on Wireless Sensor (ICWISE), Kuching, Malaysia,2013: 36-41 .

[8] L. Qiong and Y. Jun, " An improved AODV Protocol of wireless sensor network", in Proc.of. International Conference on Image Analysis and Signal Processing (IASP), 2012.

[9] M. N. Jambli, K. Zen, H. Leonando and A. Tully, " Performance evaluation of AODV protocol for mobile wireless sensor network", in Proc. of 7th International Conference on Information Technology in Asia (CITA 11), 2011.

[10] J. Rahman, M. A. M. Hassan and M. K. B. Islam, "Comparative Analysis the performace of AODV, DSDV and DSR routing protocols in wireless sensor networks", in Proc. of 7th International Conference on Electrical \& Computer Engineering (ICECE), 2012.

[11] J. Zhang, D. Zhang, K. Xie, et al., "A Cooperative Routing Algorithm for Maximizing Network Lifetime", in Advances in Wireless Sensor Networks, Springer, 2013; 665-675. 
[12] F. Mansourkiaie and M. H. Ahmed, "Joint Cooperative Routing and Power Allocation for Collision Minimization in Wireless Sensor Networks With Multiple Flows", IEEE, Wireless Communications Letters, 2015; 4(1): 6-9.

[13] M. Dong, K. Ota, A. Liu, M. Guo, "Joint optimization of lifetime and transport delay under reliability constraint wireless sensor networks", IEEE Transactions on Parallel Distributed Systems, 2016; 27 (1): 225-236.

[14] Y. Liu, M. Dong, K. Ota and A. Liu, "ActiveTrust: Secure and Trustable Routing in Wireless Sensor Networks" IEEE Transactions on Information Forensics and Security, 2016; 11(9), 2013 - 2027.

[15] Z. Chen, A. Liu, Z. Li, Y-J Choi, H. Sekiya, and J. Li "Energy-Efficient Broadcasting Scheme for Smart Industrial Wireless Sensor Networks" Hindawi Mobile Information Systems, 2017; 1-17.

[16] N. Abdolmaleki, M. Ahmadi, H. T. Malazi, S. Milardo, "Fuzzy topology discovery protocol for SDN-based wireless sensor networks", Elsevier Simulation Modelling Practice and Theory, 2017, 79: 54-68.

[17] F. A. Aoudia, M. Gautier, and O. Berderr, "RLMan: An Energy Manager Based on Reinforcement Learning for Energy Harvesting Wireless Sensor Networks" IEEE Transactions on Green Communications and Networking, 2018; 2(2) :408-417.

[18] J. Liu, H. Shen, L. Yu, and H. S. Narman, "Characterizing Data Deliverability of Greedy Routing in Wireless Sensor Networks" IEEE Transactions on Mobile Computing, 2018; 17(3) : 543 - 559.

[19] B. Arisian and K. Eshghi, "A Game Theory Approach for Optimal Routing: In Wireless Sensor Networks", in Proc. of 6th International Conference on Wireless Communications Network and Mobile Computing (WiCOM), 2010.

[20] H. Y. Shi, W. L. Wang, N. M. Kwok and S. Y. Chen, "A Game Theory for Wireless Sensor Networks: A Survey", Journal Sensors, 2012; 9055-9097.

[21] Z. Xu, Y. Yin and J. Wang, "An Density-based Energy-efficient Routing Algorithm in Wireless Sensor Networks Using Game Theory", International Journal of Future Generation Communication and Networking, 2012, 5(4), 99112.

[22] D. Sun, X. Huang, Y. Liu and H. Zhong, “ Predictable Energy Aware Routing based on Dynamic Game Theory in Wireless Sensor Networks”, Elsevier Computers \& Electrical Engineering, 2013; 39( 6) : 1601-1608.

[23] D. Lin, Q. Wang, D. Lin, and Y. Deng, “ An Energy-Efficient Clustering Routing Protocol Based on Evolutionary Game Theory in Wireless Sensor Networks", Hindawi International Journal of Distributed Sensor Networks, 2015; $1-12$.

[24] R. Feng, T. Li, Y. Wu, and N. Yu, " Reliable routing in wireless sensor networks based on coalitional game theory", IET Communications, 2016; 10( 9): 1027-1034.

[25] D. Lin and Q. Wang, "A game theory based energy efficient clustering routing protocol for WSNs", Journal of Wireless Networks, 2017; 23 (4) :1101-1111.

[26] M. Altayeb, S. Abdalla, and M. Mekki, "Wireless Sensor Network for Radiation Detection", Indonesian Journal of Electrical Engineering and Informatics (IJEEI), 2017, 5(1): 37- 43.

[27] M. Mekki, and O. Abdallah, "Development of Wireless Sensor Network for Green Monitoring and Control", Indonesian Journal of Electrical Engineering and Informatics (IJEEI), 2017, 5(3): 270-274.

[28] M. Chincoli and A. Liotta, "Self-Learning Power Control in Wireless Sensor Networks", MDPI Journal Sensors, $2018 ; 18,375: 2-29$.

[29] S. Kassan, J. Gaber, and P. Lorentz, "Game Theory based distributed clustering approach to maximize Wireless sensors network lifetime", Journal of Network and Computer Application, 2017, 123: 80-88.

[30] I. Attiah, M. Chatterjee, and C. C. Zou, "A Game Theoretic Approach for Energy-Efficient Clustering in Wireless Sensor Networks", in Proc.of. IEEE Wireless Communications and Networking Conference (WCNC), 2017: 1-6.

[31] K. S. Adu-Manu, A. N. Adam, Tapparello, A. H. Ayatollah and A. W. Heinzelman, ' Energy-Harvesting Wireless Sensor Networks(EH WSNs): A Review, “ACM Transactions on Sensor Networks, 2018, 14(2):1-50.

[32] G. N. Basavaraj, C. D. Jaidhar, "Low latency and energy efficient cluster based routing design for wireless sensor network", Indonesian Journal of Electrical Engineering and Computer Science, 2019; 13(2): 615-625.

[33] S. A. C. Aziz, A. F Kadmin, N. Rahim, W. H. W Hassan, I. F. A Aziz, M. S. Hamid, and R. A. Hamzah, "Development of automatic healthcare instruction system via movement gesture sensor for paralysis patient", International Journal of Electrical and Computer Engineering (IJECE) 2019; 9(3) :1676 1682. 